\title{
Enhanced neuroinflammation mediated by DNA methylation of the glucocorticoid receptor triggers cognitive dysfunction after sevoflurane anesthesia in adult rats subjected to maternal separation during the neonatal period
}

Yangzi Zhu ${ }^{1,2}$, Yu Wang ${ }^{1}$, Rui Yao ${ }^{1}$, Ting Hao ${ }^{1}$, Junli Cao ${ }^{1}$, He Huang ${ }^{1}$, Liwei Wang ${ }^{2 *}$ and Yuqing Wu ${ }^{1,3^{*}}$

\begin{abstract}
Background: Mounting evidence indicates that children who experience abuse and neglect are prone to chronic diseases and premature mortality later in life. One mechanistic hypothesis for this phenomenon is that early life adversity alters the expression or functioning of the glucocorticoid receptor (GR) throughout the course of life and thereby increases sensitivity to inflammatory stimulation. An exaggerated pro-inflammatory response is generally considered to be a key cause of postoperative cognitive dysfunction (POCD). The aim of this study was to examine the effects of early life adversity on cognitive function and neuroinflammation after sevoflurane anesthesia in adult rats and to determine whether such effects are associated with the epigenetic regulation of GR.

Methods: Wistar rat pups were repeatedly subjected to infant maternal separation (early life stress) from postnatal days 2-21. In adulthood, their behavior and the signaling of hippocampal pro-inflammatory factors and nuclear factor-kappa B (NF-KB) after sevoflurane anesthesia were evaluated. We also examined the effects of maternal separation (MS) on the expression of GR and the DNA methylation status of the promoter region of exon 17 of GR and whether behavioral changes and neuroinflammation after anesthesia were reversible when the expression of GR was increased by altering DNA methylation.

Results: MS induced cognitive decline after sevoflurane inhalation in the Morris water maze and context fear conditioning tests and enhanced the release of cytokines and the activation of astrocyte intracellular NF-kB signaling induced by sevoflurane in the hippocampus of adult rats. Blocking NF-KB signaling by pyrrolidine dithiocarbamate (PDTC) inhibited the release of cytokines. MS also reduced the expression of GR and upregulated the methylation levels of the promoter region of GR exon $1_{7}$, and such effects were reversed by treatment with the histone deacetylase inhibitor trichostatin A (TSA) in adult rats. Moreover, TSA treatment in adult MS rats inhibited the overactivation of astrocyte intracellular NF-KB signaling and the release of cytokines and alleviated cognitive dysfunction after sevoflurane anesthesia.

(Continued on next page)
\end{abstract}

\footnotetext{
* Correspondence: doctorlww@sina.com; xzmcyqwu@sina.com

${ }^{2}$ Department of Anesthesiology, Xuzhou Central Hospital, 199 Jiefang South

Road, Xuzhou, People's Republic of China

'Jiangsu Province Key Laboratory of Anesthesiology, Xuzhou Medical

University, 209 Tongshan, Xuzhou 221004, People's Republic of China

Full list of author information is available at the end of the article
} 
(Continued from previous page)

Conclusions: Early life stress induces cognitive dysfunction after sevoflurane anesthesia, perhaps due to the aberrant methylation of the GR gene promoter, which reduces the expression of the GR gene and facilitates exaggerated inflammatory responses.

Keywords: Sevoflurane, Neuroinflammation, Pro-inflammatory factors, Cognitive dysfunction, Glucocorticoid receptor, DNA methylation

\section{Background}

Postoperative cognitive dysfunction (POCD) is a relatively common and well-known complication in surgical patients. The occurrence of POCD is associated with increased mortality, risk of withdrawal from the labor market, and dependency on social transfer payments $[1,2]$. However, despite enormous research efforts in recent decades, the pathogenesis of POCD remains obscure. Mounting evidence has revealed that inflammation plays a key role in the disease process [3-5]. Peripheral inflammation due to surgical trauma and the release of accompanying systemic inflammatory mediators have been shown to influence inflammatory processes of the central nervous system, triggering the activation of neurogliocytes and the concurrent endogenous production of pro-inflammatory cytokines [6-9]. Volatile anesthetics, particularly isoflurane and sevoflurane, directly increased the production of pro-inflammatory cytokines in the brains of mice and impaired the acquisition of spatial memory in aged rats [10-12]. In addition to surgical trauma and anesthetics, advanced age is one of the main risk factors for the development of POCD [13]. Aging has been associated with an exacerbated inflammatory response in the normal aged brain when the immune system is irritated [14, 15], which may increase susceptibility to POCD in the aged [16-18]. However, the occurrence of POCD exhibits pronounced individual differences in the elderly, and POCD is also observed in younger and adult patients [2].

Accumulating evidence indicates that early life adversity is associated with an increased risk for various longterm mental and physical diseases, including depression, anxiety, autoimmune disorders, diabetes, hypertension, cardiovascular diseases, cancers, and premature mortality [19-25]. The consequences of early life adversity can last a lifetime, even when individuals' life situations improve after experiencing early negative life events $[26,27]$. A hypothesis concerning the mechanism of susceptibility to chronic diseases is that early life adversity induces glucocorticoid insensitivity and exaggerates inflammatory responses to injury or infection [28]. Several studies have shown that early life adversity results in an increased methylation of the glucocorticoid receptor (GR) gene promoter and reduced GR expression in rodents and humans [29-31], which may lead to glucocorticoid feedback resistance and GR desensitization [28, 32]. It is generally accepted that GR signaling regulates the inflammatory response to noxious stimuli, and evidence has supported this hypothesis. Adult individuals who experienced early life adversity were shown to have elevated levels of C-reactive protein (CRP) and pro-inflammatory cytokines (interleukin (IL)-1, IL-6, and tumor necrosis factor (TNF)$\alpha)$ compared with participants without early life stress [33-36]. Furthermore, an animal study showed that early life stress enhanced the production of pro-inflammatory cytokines in response to viral infection [37]. Thus, will early life adversity amplify the neuroinflammation induced by volatile anesthetics or surgeries and increase the risk of POCD?

The aim of this study was to examine whether early life adversity induces cognitive decline and exaggerates neuroinflammation after sevoflurane anesthesia in adult rats. Moreover, we examined the effects of early life adversity on the expression of GR and the methylation of GR gene promoters and whether behavioral changes and neuroinflammation after anesthesia were reversible if GR expression was increased by altering DNA methylation.

\section{Methods}

\section{Maternal separation model}

All experimental procedures involving animals were approved by the Animal Care and Use Committee of Xuzhou Medical University (Xuzhou, Jiangsu Province, China). Wistar rats were obtained from the Experimental Animal Center of the Xuzhou Medical University. Male and female rats were housed together in standard rat cages, each containing one male and two females. Each female rat was housed separately after pregnancy. Animals were maintained under standard laboratory conditions, with a 12-h light/dark cycle (lights on at 6:00 a.m.), a room temperature of $23 \pm 1{ }^{\circ} \mathrm{C}$, and food and water provided ad libitum. The pups remained undisturbed at birth (postnatal day 0, P0). Half of the male pups from each litter were randomly assigned to undergo maternal separation (MS). The other half of the male pups and all female pups were kept with their dam the entire time. The separated pups were removed from the home cage and placed in a warm standard laboratory cage for $6 \mathrm{~h}$ per day (7:00-10:00 and 13:00-16:00) from P1 to P21 [38]. The pups were returned to the home 
cage and remained with their dam the rest of the time. Following weaning, all pups were group-housed at P22 (6-7 per cage) according to gender and whether they had been subjected to MS or not. We did not observe any differences in body weight between MS rats and normal rats (data not shown). Subsequent experiments were performed when the animals reached adult age (85-90 days old).

\section{Rats anesthesia and treatment}

Rats received 3\% sevoflurane mixed with pure oxygen for $2 \mathrm{~h}$ in anesthetizing chambers. Control groups received pure oxygen for $2 \mathrm{~h}$ in identical chambers. The anesthesia with $3 \%$ sevoflurane mixed with pure oxygen for $2 \mathrm{~h}$ was clinically relevant and did not cause significant changes in blood pressure and blood gas compared with the control group (data not shown). Anesthetizing chambers were placed on a heating pad, and the rat's body temperature was maintained at $37 \pm 0.5{ }^{\circ} \mathrm{C}$. The concentrations of sevoflurane and oxygen were measured continuously (Drager, Lubeck, Schleswig-Holstein, Germany). Anesthesia was terminated by discontinuing sevoflurane and by administering pure oxygen until the animal regained its righting reflex. To evaluate the role of the nuclear factor-kappa B (NF- $\mathrm{kB}$ ) signaling pathway, pyrrolidine dithiocarbamate (PDTC; $100 \mathrm{mg} / \mathrm{kg}$ ) [39], an NF- $k B$ inhibitor, was given to rats intraperitoneally $30 \mathrm{~min}$ before exposure to sevoflurane. For intervention studies, the histone deacetylase inhibitor trichostatin A (TSA) was used for the epigenetic regulation of the glucocorticoid receptor. Rats were implanted with a stainless steel guide cannula (22 gauge, RWD, China) directed toward the left lateral ventricle $(1.6 \mathrm{~mm}$ lateral to the midline, $1.0 \mathrm{~mm}$ posterior to the bregma, and $4.0 \mathrm{~mm}$ below the surface of the dura). Other procedures were performed as described in previous studies [29]. A volume of $2 \mu \mathrm{l}$ of TSA (100 $\mathrm{ng} / \mathrm{ml}$ in DMSO) was infused using a micro-syringe through the infusion cannula for at least $1 \mathrm{~min}$. Rats received a single infusion daily for seven consecutive days before exposure to sevoflurane.

\section{Morris water maze tests}

The Morris water maze (MWM) was a cylindrical, black-painted pool (1.5 $\mathrm{m}$ in diameter, $0.6 \mathrm{~m}$ in height) filled with water $\left(0.3 \mathrm{~m}\right.$ deep, $\left.24 \pm 1{ }^{\circ} \mathrm{C}\right)$ and divided into four virtual quadrants, with one starting point in each quadrant. A black-painted platform $(10 \mathrm{~cm}$ diameter, $1 \mathrm{~cm}$ below water surface) was placed in the determinate quadrant. The experimental room contained cues that remained unchanged throughout the study. The movements of the rats were recorded by a video-tracking/ computer-digitizing system (Shanghai Jiliang Software Technology Co., Ltd., Shanghai, China). The P86 rats were tested in the MWM four times per day for 4 days (acquisition tests). Each rat was gently placed in the water at one of the four starting points (in a random order) along the water maze perimeter with its face toward the wall of the pool. Rats were given $120 \mathrm{~s}$ to find the platform and were then left on the platform for $30 \mathrm{~s}$. If the rat did not find the hidden platform within $120 \mathrm{~s}$, the researcher would gently guide it to the platform, and its escape latency to find the platform was then marked as $120 \mathrm{~s}$. At the end of the MWM training (P90), the platform was removed and the animals were allowed to search in the pool for $120 \mathrm{~s}$ (probe test); the number of crossings over the target area and the search time in the target quadrant were recorded [40]. Animals that performed MWM tests were not used in other tests.

\section{Context fear conditioning tests}

Fear conditioning experiments were performed in an acrylic conditioning chamber with a grid floor composed of 19 stainless steel bars. A ventilation fan supplied background noise $(65 \mathrm{db})$, and overhead infrared lights were left on. An infrared camera was located on the walls to monitor the rats' freezing behavior. P86 rats were given $5 \mathrm{~min}$ to acclimate to the chamber and were then presented with one tone $(2.2 \mathrm{kHz}$ and $96 \mathrm{~dB}$ for $30 \mathrm{~s})$. During the last $2 \mathrm{~s}$ of the tone, the rats were given a single shock $(2.0 \mathrm{~mA}, 2 \mathrm{~s})$. After the shock, the rats stayed in the chamber for $30 \mathrm{~s}$ and were then placed back into their housing area. Memory retention was assessed at $48 \mathrm{~h}$ post-conditioning. P88 rats were returned to the original chamber and allowed to stay there for $3 \mathrm{~min}$. The chamber was maintained in the same context as when the rats were conditioned but without a footshock or tone. Freezing time during this period was recorded as a measure of contextual fear memory [41]. Animals that underwent context fear conditioning tests were not used in other tests.

\section{Hippocampus tissue preparation}

Immediately after undergoing anesthesia with $10 \%$ chloral hydrate $(0.3 \mathrm{ml} / 100 \mathrm{~g}$, i.p.), all animals were sacrificed by decapitation. The hippocampus was rapidly removed, frozen in liquid nitrogen, and then stored at $-80{ }^{\circ} \mathrm{C}$ until further processing. For enzyme-linked immunosorbent assay and Western blot analysis, the harvested hippocampus tissues were homogenized on ice using radioimmunoprecipitation assay (RIPA) lysis buffer supplemented with protease inhibitors (Beyotime Institute of Biotechnology, Haimen, Jiangsu, China). The lysates were collected and centrifuged at 12,000 rpm for $15 \mathrm{~min}$ to extract total proteins. The EpiQuik Nuclear Extraction Kit (Epigentek, Brooklyn, NY, USA) was used for the preparation of nucleoproteins 
from the hippocampal tissues according to the manufacturer's instructions. The supernatant was quantified for total protein using the bicinchoninic acid (BCA) protein assay kit (Beyotime Institute of Biotechnology, Haimen, Jiangsu, China).

\section{Western blot analysis}

Western blotting was used to determine the expression of GR in the total protein extract and NF- $\mathrm{kB}$ p65 in the nuclear extract from the hippocampal tissues. Equal amounts of protein were loaded and separated by sodium dodecyl sulfate-polyacrylamide gel electrophoresis (SDS-PAGE) and transferred to nitrocellulose membranes (Millipore Corporation, Billerica, MA). The membranes were blocked in 5\% nonfat milk for $2 \mathrm{~h}$ at room temperature and then incubated overnight at $4{ }^{\circ} \mathrm{C}$ with rabbit anti-GR (1:200, Santa Cruz Biotechnology, CA, USA), rabbit anti-NF-kB p65 (1:500, Abcam, Cambridge, UK), and mouse anti- $\beta$-actin (1:1000, SigmaAldrich). After the incubation, the membranes were washed three times with phosphate-buffered saline (PBS, $\mathrm{pH}$ 7.4) containing $0.3 \%$ Triton $\mathrm{X}-100$ (PBS-T) and incubated with corresponding secondary antibodies conjugated with horseradish peroxidase (1:500, ZSGB-BIO, Beijing, China) for $2 \mathrm{~h}$ at room temperature. The protein signals were finally visualized using an enhanced BCIP/ NBT Alkaline Phosphatase Color Development Kit (Beyotime Biotechnology, Inc., Haimen, Jiangsu, China).

\section{Enzyme-linked immunosorbent assay}

TNF- $\alpha$, IL- $1 \beta$, and IL- 6 levels were quantified using ratspecific enzyme-linked immunosorbent assay (ELISA) kits (Xitang Bio-tech. Co., Ltd, Shanghai, China) according to the manufacturer's instructions. The optical density of each well was determined using a microplate reader at $450 \mathrm{~nm}$. Cytokine concentrations were calculated using standard curves generated using recombinant TNF- $\alpha$, IL-1 $\beta$, and IL-6.

\section{Immunofluorescent staining}

Rats were anesthetized with $10 \%$ chloral hydrate $(0.3 \mathrm{ml} /$ $100 \mathrm{~g}$, i.p.) and perfused transcardially with $200 \mathrm{ml}$ of $0.9 \%$ saline followed by $300 \mathrm{ml}$ of $4 \%$ paraformaldehyde in $0.1 \mathrm{M}$ phosphate buffer ( $\mathrm{pH}$ 7.4). The brain tissues were removed and postfixed in $4 \%$ paraformaldehyde overnight and cryoprotected in $30 \%$ sucrose. Thirtymicron-thick frozen sections from the rat brains were cut using a freezing microtome and serially collected throughout the hippocampus. Free-floating tissue sections were rinsed three times with PBS-T. The tissue sections were incubated with $10 \%$ normal donkey serum in PBS-T for $2 \mathrm{~h}$, followed by incubation with rabbit anti-p-NF-kB p65 (1:500, Cell Signaling Technology, Inc., Beverly, MA) and mouse anti-glial fibrillary acidic protein (GFAP) monoclonal antibody (1:600, Abcam, Cambridge, UK) at $4{ }^{\circ} \mathrm{C}$ for $24 \mathrm{~h}$. After three 5 -min rinses in PBS, the sections were incubated with donkey anti-rabbit IgG conjugated to Alexa Fluor 488 and donkey anti-mouse IgG conjugated to Alexa Fluor ${ }^{\circ} 594$ (1:500, Life Technologies, Carlsbad, CA, USA) in the dark for $2 \mathrm{~h}$ at $37{ }^{\circ} \mathrm{C}$. Fluorescence intensity was visualized under a confocal microscope (FV1000, Olympus Corp., Tokyo, Japan). The intensity on four slides (three to four sections per slide) was averaged for each animal and then normalized by that of the control group.

\section{Quantitative reverse transcriptase polymerase chain reaction}

Quantitative reverse transcriptase polymerase chain reaction (qRT-PCR) was used to assess the mRNA levels of GR in rat hippocampal tissue. Total RNA was extracted using a TRIzol reagent kit (Invitrogen, USA) and transcribed into cDNA using a high-capacity cDNA reverse transcription kit (Applied Biosystems, Foster City, USA). The PCR primers for GR were 5'-TAGGTGGGCGTC AAGTGATT-3' (forward) and 5'-GATCAGGAGCAA AGCAGAGC-3' (reverse). The primers for GAPDH were 5' -CAAGGTCATCCATGACAACTTTG-3' (forward) and 5'-GTCCACCACCCTGTTGCTGTAG-3' (reverse). Real-time PCR analysis was performed using the Roche LightCycler 480 detection system using a SYBR $^{\circ}$ Select Master Mix Kit (Life Technologies, Carlsbad, CA, USA). The relative expression levels of GR were normalized to GAPDH.

\section{DNA methylation assay}

DNA was extracted from the hippocampal tissue using the genome extraction kit (Generay Biotechnology, Shanghai, China). Sodium bisulfite modification of DNA was performed using the EpiTect Bisulfite kit (Qiagen, Venlo, Netherlands) according to the manufacturer's protocol. PCR amplification was carried out in a 50- $\mu$ l reaction volume containing $25 \mu \mathrm{l}$ of PCR master mix (Qiagen, Germany), $2 \mu \mathrm{l}$ of each primer, $2.5 \mu \mathrm{l}$ of bisulfite-modified DNA, and $\mathrm{H}_{2} \mathrm{O}$. The thermocycler protocol was as follows: a 4-min denaturation at $95{ }^{\circ} \mathrm{C}$; 40 cycles of 30 -s denaturation at $95{ }^{\circ} \mathrm{C}$, a 30 -s annealing period at $55{ }^{\circ} \mathrm{C}$, and a 40 -s extension at $72{ }^{\circ} \mathrm{C}$; and a final 5-min extension at $72{ }^{\circ} \mathrm{C}$. The PCR product (285 bp) was used as a template for subsequent nested PCR reactions. The PCR product $(177 \mathrm{bp})$ was purified and separated on a $2 \%$ agarose gel. The targeted DNA fragment was extracted from the gel. Following elution in Tris buffer, the PCR product was then subcloned into the pTG19-T vector (Generay Biotechnology) according to the manufacturer's protocol. Ten plasmids containing the exon $1_{7}$ GR promoter DNA fragment were screened and sequenced per animal. 


\section{Statistical analysis}

All of the data are presented as the mean \pm SD of independent experiments. For the escape latency data, repeated-measures two-way ANOVAs between groups were conducted. The post hoc Bonferroni test was used to compare the differences in escape latency between groups on each day of the MWM. One-way ANOVA followed by the Student-Newman-Keuls post hoc test or Student's two-sample $t$ test was performed for the other behavioral tests, Western blotting, ELISA, and methylation of all CpG sites within exon $1_{7}$ of GR. Statistical analysis was performed using SPSS 16.0 (SPSS, Chicago IL) or GraphPad Prism 5.0 for Windows
(GraphPad Software, Inc., San Diego, CA). $P$ values $<0.05$ were considered to be statistically significant.

\section{Results}

Sevoflurane anesthesia induced cognitive impairment in adult maternal separation rats

To elucidate the effect of MS on cognitive function after sevoflurane inhalation in adult rats, we conducted MWM tests and context fear conditioning (CFC) tests, which are widely used to evaluate hippocampus-dependent spatial reference learning and memory in rodents [42, 43]. A schematic illustration of the experimental timeline is shown in Fig. 1a. The comparison of the time that each rat
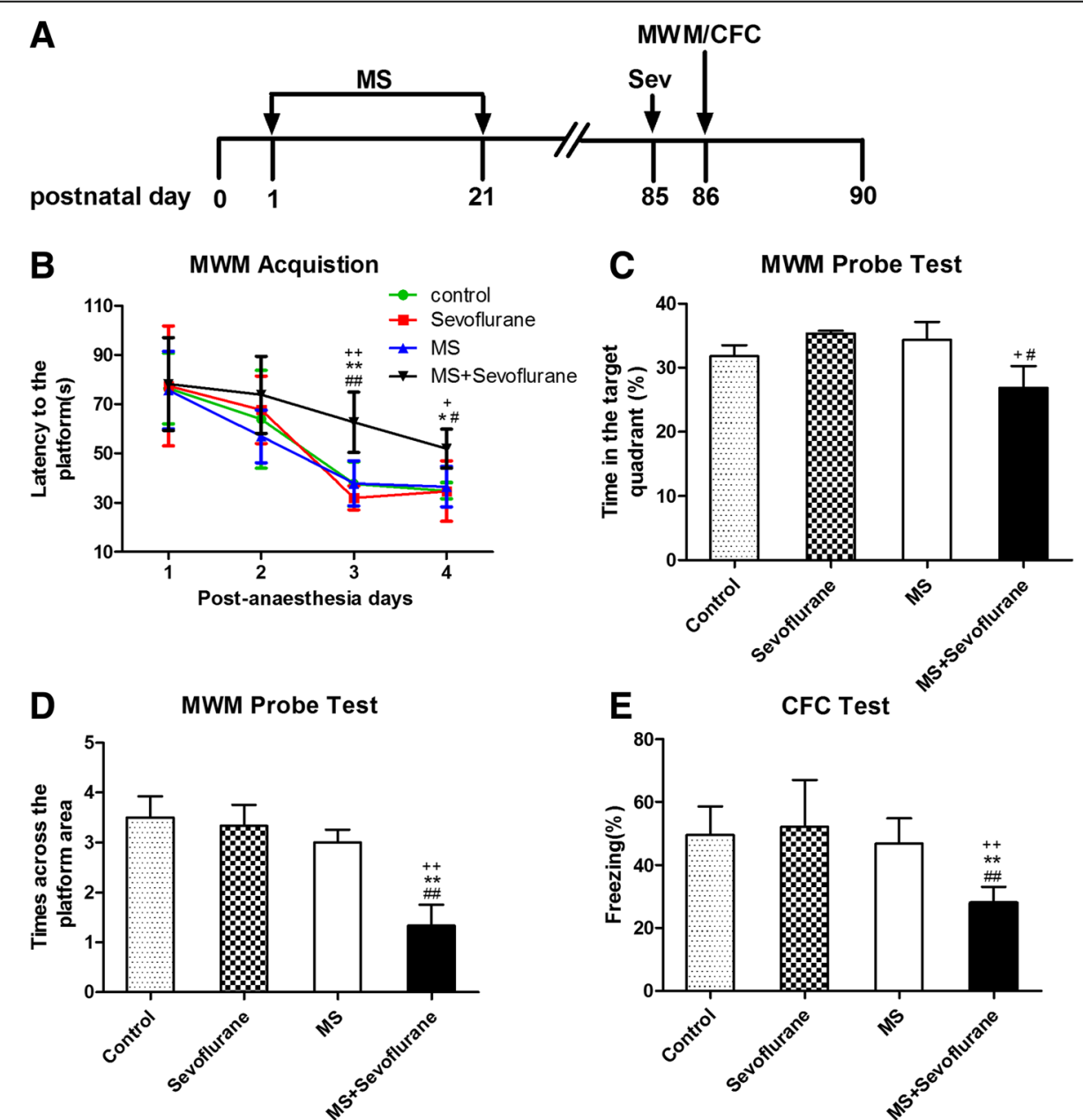

Fig. 1 The effect of neonatal maternal separation on cognitive function after sevoflurane anesthesia in adult rats. a Schematic illustration of the experimental timeline. MS maternal separation, Sev anesthesia with 3\% sevoflurane for $2 \mathrm{~h}$, MWM Morris water maze, CFC context fear conditioning. b Anesthesia with $3 \%$ sevoflurane for $2 \mathrm{~h}$ in MS rats increased the escape latency in the acquisition phase of the MWM test on the third and fourth days after sevoflurane anesthesia. There was no statistically significant interaction of time and group between groups. Analysis was performed with repeated-measures two-way ANOVA. c The time in the target quadrant in the MS + sevoflurane group was less than those in the sevoflurane group and MS group by the MWM probe test. $\mathbf{d}$ The number of crossings of the platform area was lower in the MS + sevoflurane group than in the other three groups in the MWM probe test. e MS rats subjected to sevoflurane anesthesia exhibited reduced freezing to context when compared with normal rats that received sevoflurane anesthesia and MS rats without anesthesia. ${ }^{*} P<0.05,{ }^{* *} P<0.01$ versus control; ${ }^{+} P<0.05,{ }^{++} P<0.01$ versus sevoflurane; ${ }^{P} P<0.05, \# P<0.01$ versus MS. Error bars represent the means \pm SD $(n=10)$. Statistical analyses were performed using a one-way ANOVA followed by Student-Newman-Keuls post hoc test 
took to reach the platform during reference training (escape latency) showed that there was no statistically significant interaction of time and group between groups in the acquisition phase of the MWM tests $(F=1.567, P=0.145)$. The factors of days $(F=29.488$, $P<0.001)$ and groups $(F=4.714, P=0.011)$ were statistically significant. The post hoc Bonferroni test showed that the MS rats that received sevoflurane anesthesia had a longer escape latency than the normal rats; the normal rats that received sevoflurane anesthesia and the MS rats without anesthesia on the third or fourth days after sevoflurane anesthesia (Fig. 1b; post-anesthesia day 3, $P=0.001 \mathrm{MS}+$ sevoflurane versus control, $P<0.001$ $\mathrm{MS}+$ sevoflurane versus sevoflurane, and $P=0.001 \mathrm{MS}+$ sevoflurane versus MS; post-anesthesia day $4, P=0.014$ MS + sevoflurane versus control, $P=0.013 \mathrm{MS}+$ sevoflurane versus sevoflurane, and $P=0.030 \mathrm{MS}+$ sevoflurane versus MS). Reference memory was assessed using the MWM probe test on the fifth day. The time spent in the target quadrant in the MS + sevoflurane group was less than that in MS group and sevoflurane group (Fig. 1c; $P=0.020 \mathrm{MS}+$ sevoflurane versus sevoflurane and $P=0.037 \mathrm{MS}+$ sevoflurane versus $\mathrm{MS}$ ). In addition, the number of crossings of the platform area was lower in the MS + sevoflurane group than in the other groups (Fig. 1d; $P=0.001 \mathrm{MS}+$ sevoflurane versus control, $P=0.002 \mathrm{MS}+$ sevoflurane versus sevoflurane, and $P=0.007$ MS + sevoflurane versus MS). MS rats showed a significant cognitive decline after sevoflurane anesthesia in MWM tests. The results of the CFC tests were similar to those of the MWM tests; MS rats that received sevoflurane anesthesia exhibited reduced immobility (freezing) in the CFC test (Fig. 1e; $P=0.001 \mathrm{MS}+$ sevoflurane versus control, $P<0.001 \mathrm{MS}+$ sevoflurane versus sevoflurane, and $P=0.004 \mathrm{MS}+$ sevoflurane versus MS). No statistically significant differences were observed among the control group, sevoflurane group, and MS group in the MWM and CFC tests.

\section{Maternal separation enhanced the release of cytokines and activation of astrocytes and NF-KB signaling induced by sevoflurane in the hippocampus}

The above findings suggested that sevoflurane anesthesia in MS rats might induce cognitive impairment; therefore, we continued to investigate the underlying mechanisms. It has been reported that pro-inflammatory cytokines, such as TNF- $\alpha$, IL- $1 \beta$, and IL- 6 , are associated with cognitive impairment [6-9]. Therefore, we detected the levels of TNF- $\alpha$, IL-1 $\beta$, and IL- 6 in the hippocampi of rats after sevoflurane anesthesia. An ELISA for TNF$\alpha$ showed that 3\% sevoflurane anesthesia for $2 \mathrm{~h}$ increases the levels of TNF- $\alpha$ at $0,6,12$, and $24 \mathrm{~h}$ after anesthesia both in control and MS rats, but the levels of TNF- $\alpha$ in MS rats were markedly higher than in control rats at each time point after anesthesia (Fig. 2a). The changes in the levels of IL-1 $\beta$ and IL- 6 in the hippocampus were similar to those of TNF- $\alpha$ (Fig. 2b, c).

Previous studies have shown that inhaled anesthetic can activate the NF- $\mathrm{kB}$ signaling pathway [44], which is a core signaling pathway associated with the expression of pro-inflammatory cytokines. To examine whether the observed changes in inflammatory mediators are related to the NF- $\mathrm{kB}$ signaling pathway, we determined the expression of NF- $\mathrm{kB}$ p65 in the nuclear extracts from the hippocampal tissues. Western blot analysis showed that $3 \%$ sevoflurane anesthesia for $2 \mathrm{~h}$ increases the levels of nuclear NF-kB p65 protein at $0,6,12$, and $24 \mathrm{~h}$ after anesthesia both in control and MS rats, but the levels of nuclear NF- $\mathrm{kB}$ p65 in MS rats were markedly higher than that in control rats at each time point after anesthesia (Fig. 2d).

The glia is the main source of inflammatory cytokines in the central nervous system. It has been reported that rats with POCD had increased levels of pro-inflammatory cytokines and astrocyte activation [9]. Activation of the astroglial NF- $\mathrm{kB}$ signaling pathway can regulate neuroinflammation because it drives the transcription of several pro-inflammatory molecules $[45,46]$. We therefore assessed the effects of sevoflurane anesthesia on astrocytes and the astroglial NF- $\mathrm{kB}$ signaling pathway in the hippocampus between control and MS rats. GFAP immunofluorescence was increased $12 \mathrm{~h}$ after anesthesia in both the sevoflurane group and the MS + sevoflurane groups (CA1, $P=0.040$ sevoflurane versus control, $P<0.001 \mathrm{MS}$ + sevoflurane versus MS; DG, $P=0.008$ sevoflurane versus control, $P<0.001 \mathrm{MS}+$ sevoflurane versus MS). The fluorescence intensity of GFAP in MS + sevoflurane group rats was markedly higher than that in sevoflurane group rats (Fig. 3a, b; CA1, $P<0.001 \mathrm{MS}+$ sevoflurane versus sevoflurane; DG, $P=0.003 \mathrm{MS}+$ sevoflurane versus sevoflurane). A similar change in the pattern was seen with phosphorylated nuclear factor-kappa B (p-NF- $\mathrm{B}$ p65) (Fig. 3a, b). We also found that p-NF-kB p65 mainly colocalized with GFAP in astrocytes (Fig. 3a, b) but not in microglia in the hippocampus (data not shown), as revealed by double immunofluorescence staining.

\section{PDTC suppresses the enhancement effect of maternal separation on NF-кB Activation and the release of pro- inflammatory cytokines induced by sevoflurane in the hippocampus}

To determine whether the NF- $k B$ signaling pathway was involved in the increase of neuroinflammation after sevoflurane anesthesia in MS rats, we evaluated the effect of the NF- $\mathrm{kB}$ inhibitor PDTC on NF- $\mathrm{kB}$ activation and the release of pro-inflammatory cytokines $12 \mathrm{~h}$ after anesthesia in MS rats. Western blot analysis showed that PDTC pretreatment significantly prevented the enhancement effect of MS on 

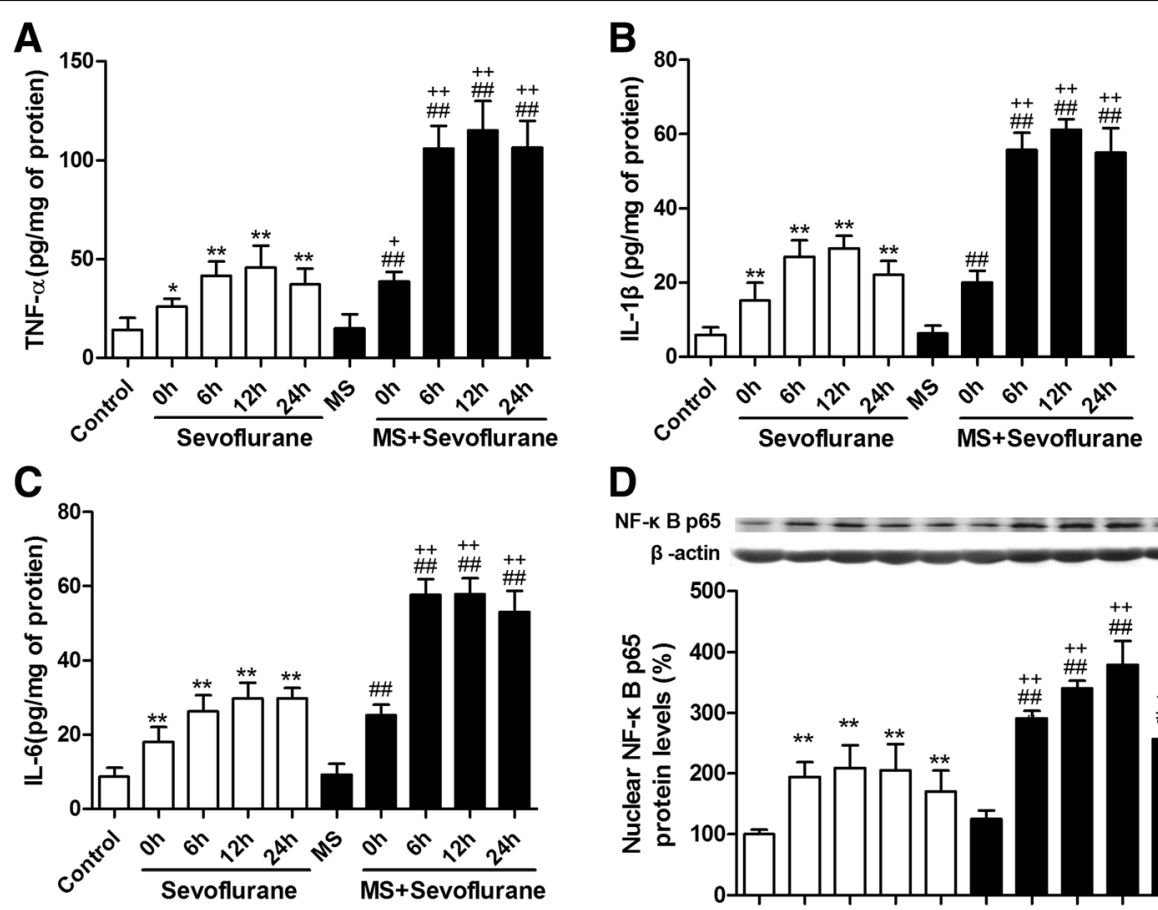

D

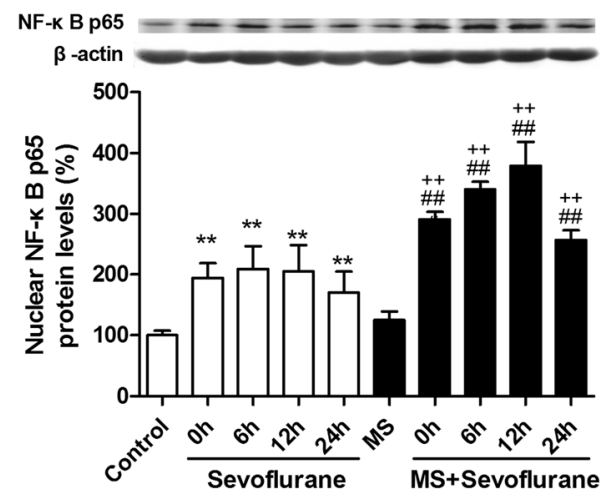

Fig. 2 MS enhanced the release of cytokines and the expression of nuclear NF-kB p65 induced by sevoflurane in the hippocampus. a Sevoflurane anesthesia for $2 \mathrm{~h}$ increased the levels of TNF- $a$ at $0,6,12$, and $24 \mathrm{~h}$ after anesthesia in both control and MS rats. The levels of TNF- $\mathrm{a}$ in MS rats were higher than that in control rats at each time point after anesthesia. The changes in the levels of IL-1 3 (b), IL-6 (c), and nuclear NF-KB p65 (d) in the hippocampus were similar to those of TNF-a. ${ }^{*} P<0.05,{ }^{* *} P<0.01$ versus control; ${ }^{\# \#} P<0.01$ versus MS; ${ }^{+} P<0.05$, ${ }^{++} P<0.01 \mathrm{MS}+$ sevoflurane versus sevoflurane (at the same time point). Error bars represent the means \pm SD $(n=6)$. Statistical analyses were performed by one-way ANOVA followed by Student-Newman-Keuls post hoc test

the levels of nuclear NF-kB p65 protein in MS rats (Fig. 4a; $t=11.03, P<0.001$ PDTC versus saline). Accordingly, an ELISA showed that PDTC also markedly suppressed the increase in hippocampal TNF- $\alpha$, IL-1 $\beta$, and IL-6 levels (Fig. 4b-d; TNF- $\alpha, t=11.05, P<0.001$ PDTC versus saline; IL-1 $\beta, t=14.98, P<0.001$ PDTC versus saline; IL-6, $t=14.57, P<0.001$ PDTC versus saline). These data suggest that the abnormal activation of the NF- $\mathrm{kB}$ signaling pathway induced by sevoflurane in MS rats may play an important role in exaggerated neuroinflammation.

Maternal separation alters the DNA methylation status of exon $1_{7}$ of the GR promoter region and decreases the expression of GR

Why do MS rats exhibit unusually excessive activation of NF- $\mathrm{kB}$ signaling and exaggerated neuroinflammation after sevoflurane anesthesia? The interaction of GRmediated signaling with NF- $\mathrm{kB}$ signaling plays a key role in the regulation of inflammatory responses. It has been reported that early life adversity (such as less maternal care and maltreatment) induces GR desensitization and exaggerates inflammatory responses to injury or infection
[28]. We therefore tested the effects of MS on the expression of GR in adult rats. Western blot analysis showed that MS decreased the expression levels of GR proteins in the hippocampus (Fig. 5a; $t=4.508, P=0.006 \mathrm{MS}$ versus control). MS-induced decreases of GR levels may be due to decreases in the generation or increases in the degradation of GR. We therefore assessed the effects of MS on the mRNA levels of GR. qRT-PCR analysis showed that MS decreased the mRNA levels of GR in the hippocampi of adult rats (Fig. $5 \mathrm{~b} ; t=4.144, P=0.002 \mathrm{MS}$ versus control). To determine whether the DNA methylation of the GR promoter alters the expression levels of GR, we tested the methylation status of $17 \mathrm{CpG}$ sites within the exon $1_{7}$ GR promoter region from the hippocampal tissue in adult rats. (Fig. 5c). Methylation analysis showed that MS rats have a higher percentage of methylated clones than control rats at most of the $17 \mathrm{CpG}$ dinucleotides in the exon $1_{7}$ GR promoter region (Fig. $5 \mathrm{~d}$ ).

\section{Trichostatin A reverses epigenetic alterations and} changes in GR expression mediated by MS

The above findings suggest that MS influences hippocampal GR expression, possibly through epigenetic 

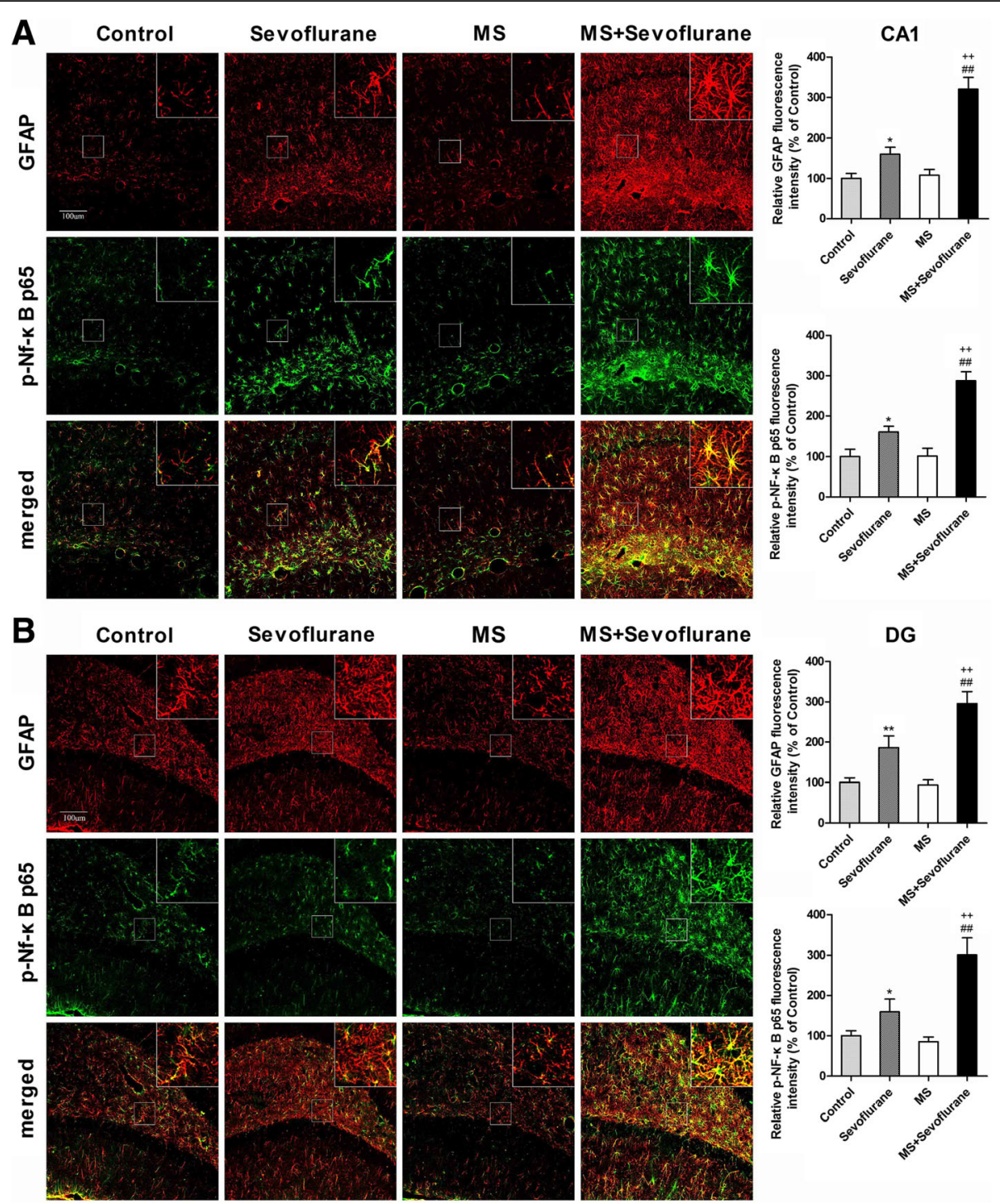

Fig. 3 MS enhanced the activation of astrocytes and the astroglial NF-KB signaling pathway induced by 3\% sevoflurane anesthesia for $2 \mathrm{~h}$ in the hippocampus. a Sevoflurane anesthesia increased the fluorescence intensity of glial fibrillary acidic protein (GFAP) and phosphorylated nuclear factor-kappa B (p-NF-kB p65) in the CA1 area of the hippocampus at $12 \mathrm{~h}$ after anesthesia in both the sevoflurane group and MS + sevoflurane group, and the fluorescence intensity of GFAP and p-NF-KB p65 in the MS + sevoflurane group was obviously higher than that in the sevoflurane group. Double immunofluorescence staining showed that p-NF-kB p65 (green) mainly co-localized (merged) with GFAP-positive reactive astrocytes (red) in the CA1 area of the hippocampus. $\mathbf{b}$ The changes in the fluorescence intensity of GFAP and p-NF-KB p65 in the DG area of the hippocampus were similar to those in the CA1 area of the hippocampus. ${ }^{*} P<0.05,{ }^{* * *} P<0.01$ versus control; ${ }^{\# \#} P<0.01$ versus MS; ${ }^{++} P<0.01$ MS + sevoflurane versus sevoflurane. Error bars represent the means \pm SD $(n=6)$. Statistical analyses were performed by one-way ANOVA followed by Student-Newman-Keuls post hoc test. CA1 cornu ammonis 1, DG dentate gyrus; bar $=100 \mu \mathrm{m}$

alterations. We therefore investigated whether the impact of MS is reversible by altering DNA methylation in adults. Previous research has shown that the histone deacetylase inhibitor TSA may induce the replicationindependent demethylation of ectopically methylated genes by increasing histone acetylation. We therefore tested the effects of TSA on the methylation status of the exon $1_{7}$ GR promoter and the expression of GR in adult MS rats. Methylation analysis of the $17 \mathrm{CpG}$ dinucleotides of the exon $1_{7}$ GR promoter showed that TSA decreased the percentage of methylated clones relative to the vehicle treatment in adult MS rats (Fig. 6a). qRT-PCR analysis showed that TSA increased the mRNA levels of GR in the hippocampi of MS rats (Fig. 6b; $t=5.230, \quad P<0.001$ DMSO versus TSA). Western blot analysis showed that TSA increased the levels of GR proteins in the hippocampi of MS rats (Fig. 6c; $t=3.592, P=0.008$ DMSO versus TSA). 

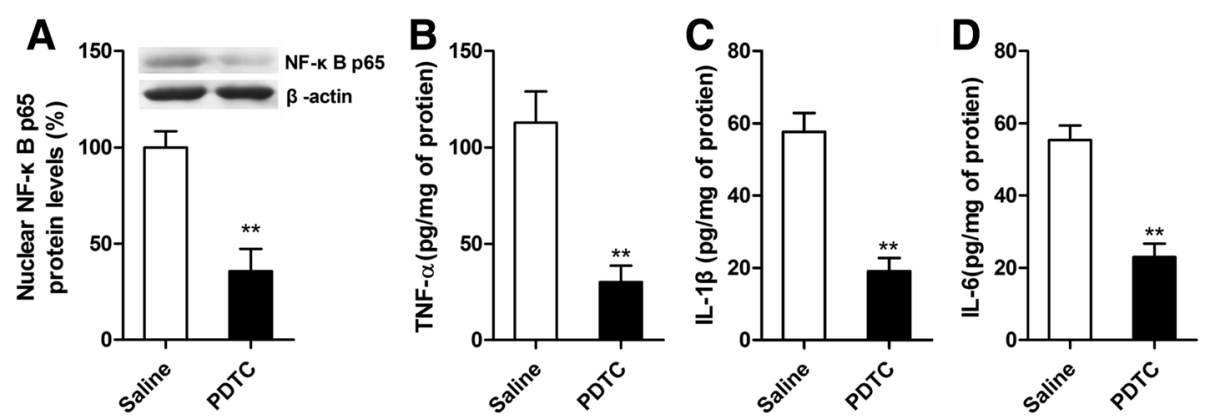

Fig. 4 PDTC inhibits the enhancement effect of maternal separation on NF-KB activation and the release of pro-inflammatory cytokines induced by sevoflurane in MS rats. PDTC or vehicle (saline) was given to rats intraperitoneally $30 \mathrm{~min}$ before exposure to sevoflurane. a PDTC pretreatment decreased the nuclear NF-KB p65 protein levels in the hippocampus $12 \mathrm{~h}$ after sevoflurane anesthesia. $\mathbf{b}$ PDTC pretreatment decreased TNF-a levels in the hippocampus $12 \mathrm{~h}$ after sevoflurane anesthesia. c PDTC pretreatment decreased IL-1 $\beta$ levels in the hippocampus $12 \mathrm{~h}$ after sevoflurane anesthesia. d PDTC pretreatment decreased IL-6 levels in the hippocampus $12 \mathrm{~h}$ after sevoflurane anesthesia. ${ }^{* *} \mathrm{P}<0.01$ versus saline. Error bars represent the means \pm SD $(n=6)$. Statistical analyses were performed with Student's two-sample $t$ test

TSA reversed the effect of MS on the activation of NF-KB signaling and neuroinflammatory responses to sevoflurane

The effects of MS on the activation of NF-kB signaling and neuroinflammatory responses to sevoflurane seem to be partly associated with changes in the hippocampal GR levels. Given that TSA treatment reversed the hippocampal expression of GR in adult MS rats, we tested the activation of NF- $\mathrm{kB}$ signaling and neuroinflammatory responses to sevoflurane in TSA - and vehicle-treated MS rats. A volume of $2 \mu \mathrm{l}$ of TSA (100 ng/ml in DMSO) was infused into the lateral ventricle of MS rats for seven consecutive days before exposure to sevoflurane. Western blot analysis showed that TSA pretreatment significantly prevented the enhancement effect of MS on the levels of nuclear NF-kB p65 protein $12 \mathrm{~h}$ after anesthesia in MS rats (Fig. 7a; $t=7.060, P<0.001$ DMSO versus TSA). An ELISA showed that TSA pretreatment also markedly suppressed the increase in hippocampal TNF- $\alpha$, IL-1 $\beta$, and IL- 6 levels $12 \mathrm{~h}$ after anesthesia in adult MS rats (Fig. 7b-d; TNF- $\alpha, t=8.564, P<0.001$ DMSO versus TSA; IL- $1 \beta, t=6.954, P<0.001$ DMSO versus TSA; IL-6, $t=3.169, P=0.001$ DMSO versus TSA). The fluorescence intensity of GFAP in the TSA group rats was markedly lower than that in the DMSO group rats at $12 \mathrm{~h}$ after anesthesia (Fig. 7e, f; CA1, $t=3.581, P=0.005$ DMSO versus TSA; DG, $t=4.188, P=0.002$ DMSO versus TSA). A similar change in pattern was observed for phosphorylated NF-kB (p-NF-kB p65) (Fig. 7e, f).

TSA attenuated the sevoflurane-induced cognitive impairment in adult maternal separation rats

Finally, we evaluated whether the reversal of the maternal effect on GR expression and neuroinflammatory responses to sevoflurane could attenuate sevofluraneinduced cognitive impairment. TSA was infused into the lateral ventricle of MS rats for seven consecutive days
(P78-P84) before exposure to sevoflurane. We conducted the MWM (P86-P90) and CFC tests (P86-P88) $24 \mathrm{~h}$ after sevoflurane anesthesia. A schematic illustration of the experimental timeline is shown in Fig. 8a. The comparison of the escape latency showed that there was a statistically significant interaction between time and group between the different groups in the acquisition phase of the MWM test according to repeated-measures two-way ANOVA $(F=5.926, P=0.035)$. The factors of days $(F=27.439, P<0.001)$ and groups $(F=6.441, P=0.029)$ were statistically significant. The post hoc Bonferroni test showed that the DMSO group rats had a longer escape latency than the TSA group rats on the third and fourth days after sevoflurane anesthesia (P88 and P89) (Fig. 8b; post-anesthesia day 3, $P=0.003$ DMSO versus TSA; postanesthesia day $4, P=0.011$ DMSO versus TSA). The time in the target quadrant in the DMSO group rats was less than that in the TSA group rats (Fig. $8 \mathrm{c} ; t=2.875, P=0.021$ DMSO versus TSA). There was no significant difference in the number of times across the platform area between the two groups (Fig. 8d). The TSA group rats showed increased immobility (freezing) at P88 (Fig. 8e; $t=2.672, P=0.032$ DMSO versus TSA). The results of the MWM and CFC tests revealed that TSA pretreatment attenuated hippocampal-dependent memory impairment induced by sevoflurane in MS rats.

\section{Discussion}

Sevoflurane is one of the most commonly used inhalational anesthetics for general anesthesia. We first found that sevoflurane anesthesia in adult (P85) MS rats induced cognitive impairment. Moreover, we found that anesthesia with $3 \%$ sevoflurane for $2 \mathrm{~h}$ induced cognitive impairment only in MS rats and not in normal rats (Fig. 1), which suggests that sevoflurane anesthesiainduced cognitive impairment has different selectivity in different individuals. These findings provide a possible 

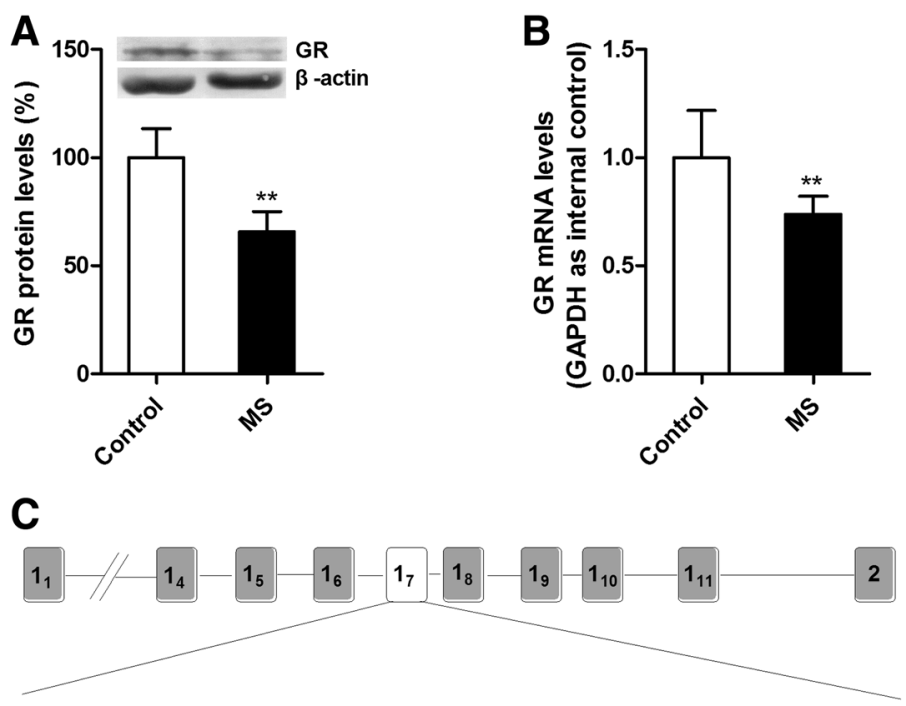

1681 ctgcccegagagcaagcggccagggctctgcggcaccgtttccgtgccatcctgtagcc 1741 ctctgctagtgtgacacacttcg ${ }^{2}$ cgcaactc ${ }^{3}$ cgcagttg ${ }^{4} \mathrm{cggg}^{5} \mathrm{cg}^{6} \mathrm{cggaccaccctg}^{7} \mathrm{c}$ 1801 ggctctg ${ }^{8}$ cggetggetgtcacccic gggggetctggctg ${ }^{10}$ cgaccca $^{11}$ cgggg $^{12}$ cgggctc $1861 \mathrm{c}^{13} \mathrm{cgag}^{14} \mathrm{cggttccaagcct}^{15} \mathrm{cggagctggg}^{16} \mathrm{cggggg}^{17} \mathrm{cgggagggagctgggagagagaaa}$

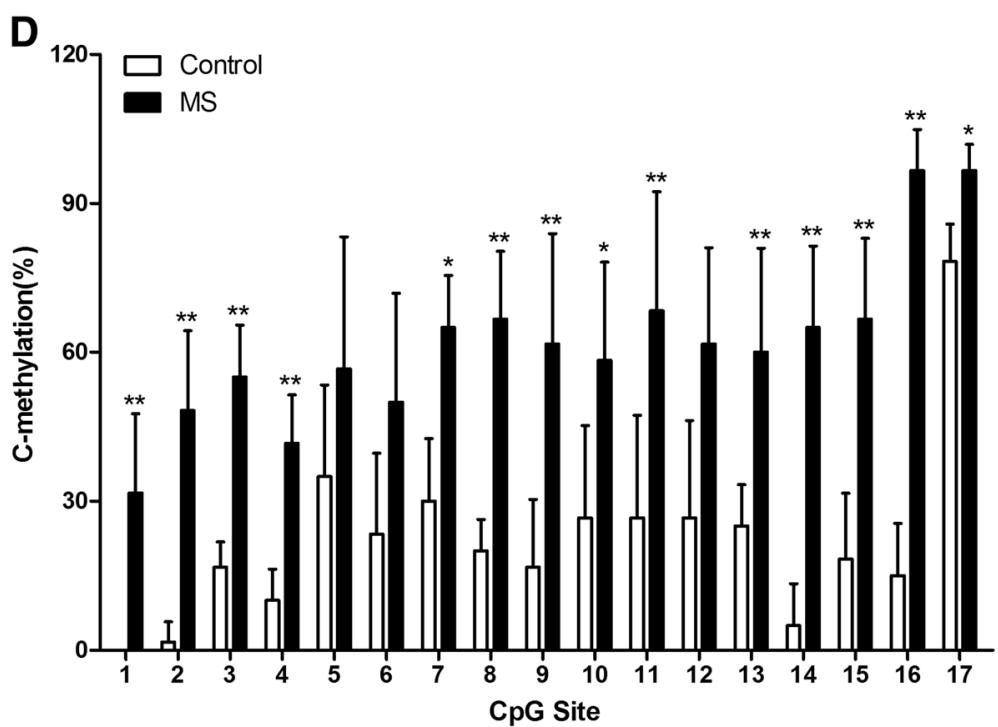

Fig. 5 Maternal separation attenuated the expression of GR and increased the percentage of methylated clones within the exon $1_{7}$ glucocorticoid receptor promoter region from the hippocampal tissues of adult rats. a GR protein levels in the hippocampal tissues of MS rats were lower compared with those of control rats. $\mathbf{b}$ qRT-PCR (GAPDH as an internal control) showed that MS decreased the mRNA levels of GR in the hippocampus compared with the control conditions. c The complete sequence map of exon $1_{7}$ of the GR promoter region (bold), including the $17 \mathrm{CpG}$ dinucleotides (superscript numbers). $\mathbf{d}$ The frequency of methylation observed at each $\mathrm{CpG}$ site in the exon $1_{7}$ GR promoter region showed that MS rats have a higher percentage of methylated clones than control rats. ${ }^{*} P<0.05,{ }^{* *} P<0.01$ versus control. Error bars represent the means $\pm S D(n=6)$.

Statistical analyses were performed with Student's two-sample $t$ test

explanation for the clinical observation that the occurrence of POCD shows distinct individual differences and suggest that individuals who have experienced early life adversity may have a predisposition to cognitive impairment after anesthesia.

Accumulating evidence suggests a pivotal role for neuroinflammation in the POCD process. Pro-inflammatory cytokine release and astrocyte activation were associated with a decline in cognitive performance in humans and animals [6-8]. Pro-inflammatory cytokines, such as TNF$\alpha$, IL- $1 \beta$, and IL- 6 , can be released by activated astrocytes, triggering neuroinflammation and leading to cognitive dysfunction [9]. Higher concentrations of pro-inflammatory cytokines inhibit long-term potentiation and impair memory [47]. We found that $3 \%$ sevoflurane anesthesia for $2 \mathrm{~h}$ increased the levels of 

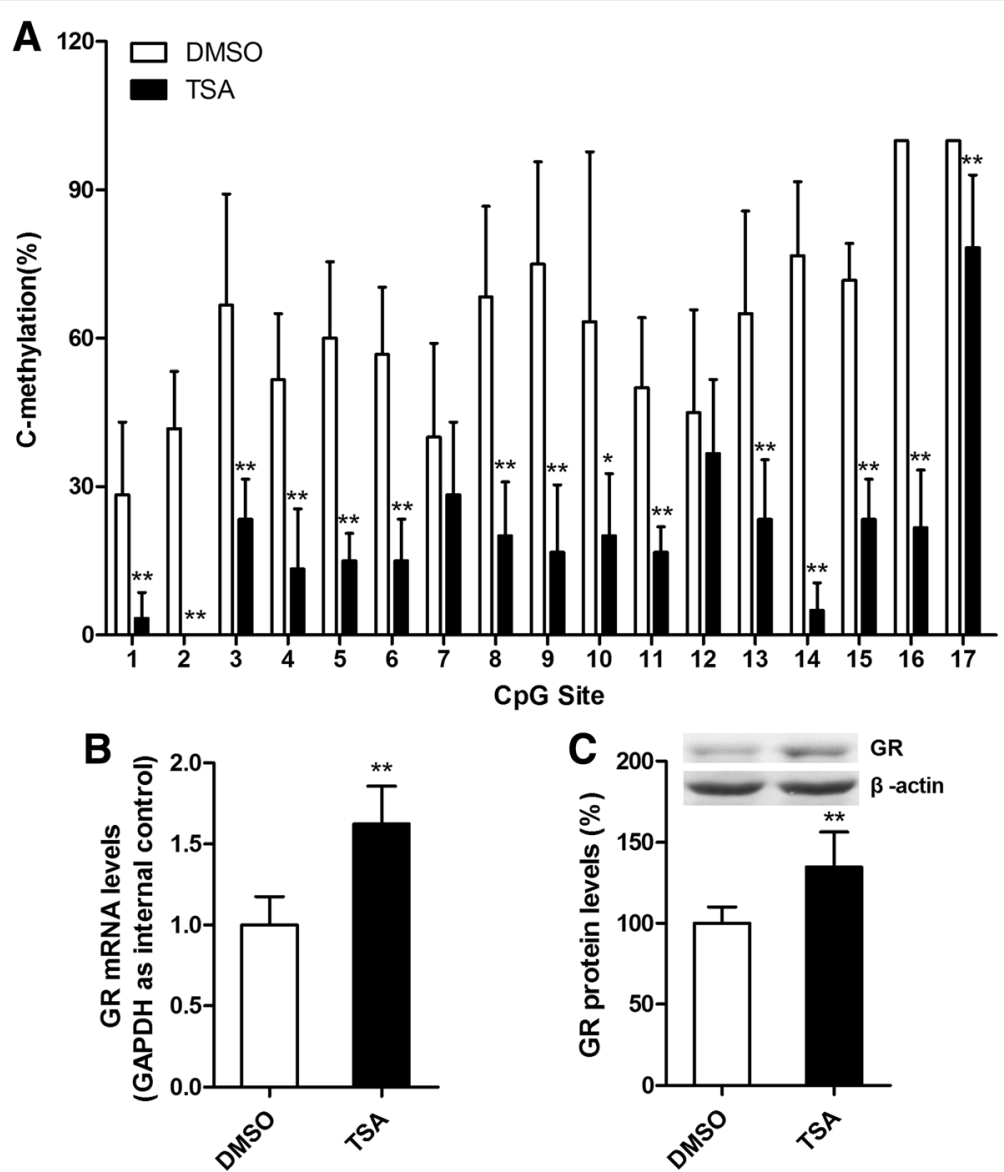

Fig. 6 TSA decreased the percentage of methylated clones within the exon $1_{7}$ GR promoter region and increased the expression of GR in the hippocampal tissues in adult MS rats. A volume of $2 \mu \mathrm{l}$ of TSA (100 ng/ml in DMSO) was infused into the lateral ventricle of MS rats for seven consecutive days. a The frequency of methylation observed at each CpG site in the exon $1_{7}$ GR promoter region showed that TSA decreased the percentage of methylated clones relative to the DMSO treatment. $\mathbf{b}$ qRT-PCR (GAPDH as internal control) showed that TSA increased the mRNA levels of GR in MS rats compared with the DMSO treatment. c TSA treatment increased the nuclear GR protein levels in the hippocampus of MS rats relative to the DMSO treatment. ${ }^{*} P<0.05,{ }^{* *} P<0.01$ versus DMSO. Error bars represent the means \pm SD $(n=6)$. Statistical analyses were performed with Student's two-sample $t$ test

TNF- $\alpha$, IL-1 $\beta$, and IL-6 in the hippocampus after anesthesia in both normal and MS rats, but the degree of proinflammatory cytokines increase in MS rats was markedly higher than that in normal rats after anesthesia (Fig. 2). These data suggest that sevoflurane anesthesia in adult MS rats may cause cognitive impairment by inducing excessive neuroinflammation. Sevoflurane anesthesia also increased GFAP (the marker of astrocyte activation) immunofluorescence in the hippocampus after anesthesia in both normal and MS rats. The degree of increase in GFAP immunofluorescence in MS rats was higher than that in normal rats, similar to the change in pro-inflammatory cytokine levels (Fig. 3). These results further suggest that rats with different early life experiences may have different neuroinflammatory reactions to sevoflurane anesthesia.

Inhalation anesthetics have been shown to activate NF- $\mathrm{B}$ signaling $[39,44]$. NF- $\kappa \mathrm{B}$ signaling plays a pivotal role in immune and inflammatory responses. Inactive
NF- $\mathrm{kB}$, a dimer of $\mathrm{p} 50$ and $\mathrm{p} 65$, remains in the cytosol. In response to diverse internal and external inflammatory stimuli, NF-kB p65 is phosphorylated and rapidly translocates to the nucleus $[48,49]$. In the current study, we found that sevoflurane anesthesia activated hippocampal NF- $k B$ signaling both in MS rats and normal rats, but the levels of nuclear NF-kB p65 protein and the fluorescence intensity of $\mathrm{p}-\mathrm{NF}-\mathrm{kB}$ p65 in the hippocampus in MS rats were significantly higher than those in normal rats (Figs. 2 and 3). These results suggest that NF- $\mathrm{KB}$ signaling in MS rats was overactivated under sevoflurane anesthesia. Next, we found that the preinhibition of NF-kB signaling by PDTC significantly suppressed excessive pro-inflammatory cytokine release after sevoflurane anesthesia in MS rats (Fig. 4). These results further suggest that the excessive activation of $\mathrm{NF}-\mathrm{kB}$ signaling was involved in the increase of neuroinflammation after sevoflurane anesthesia in MS rats. 


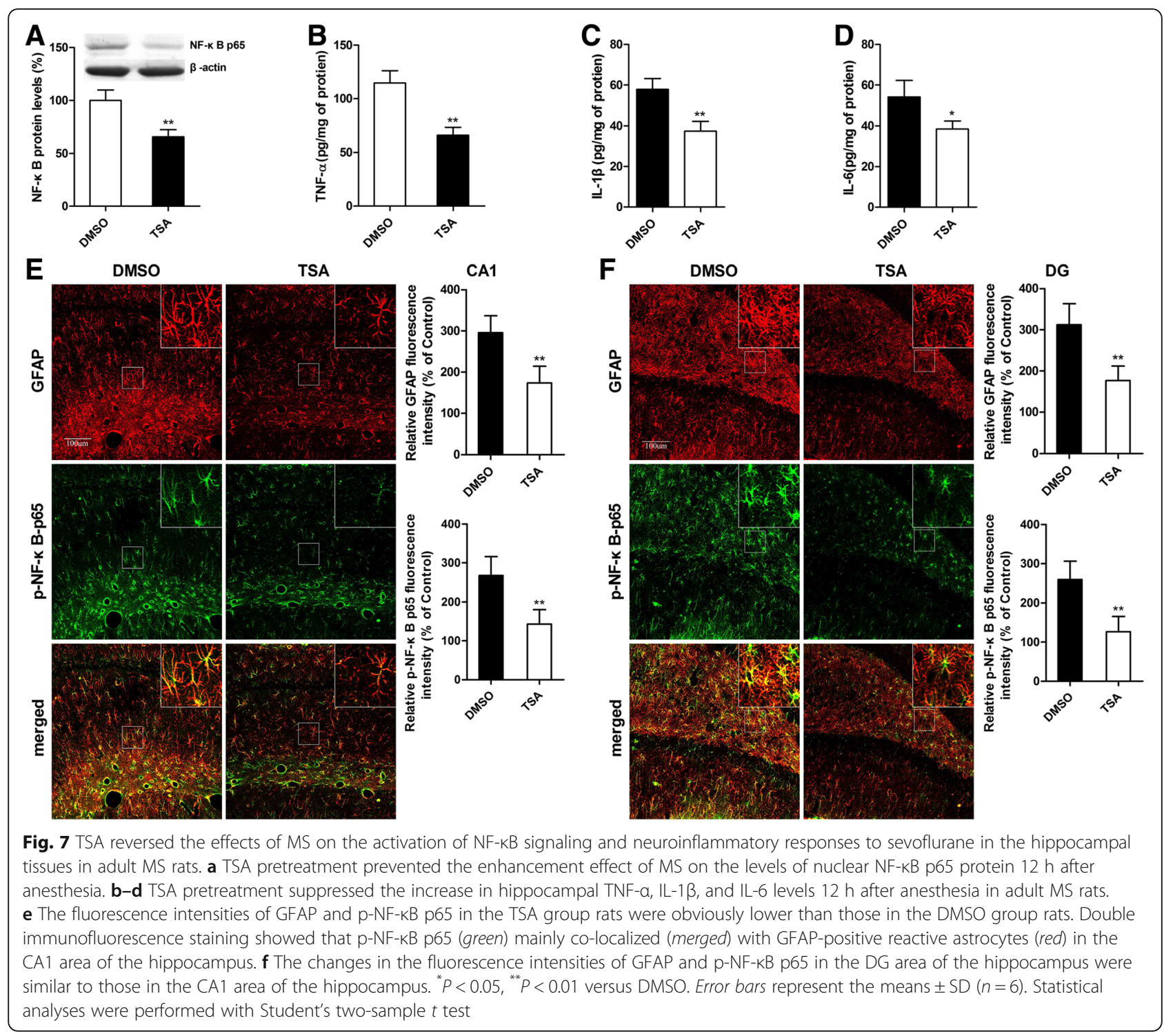

Given that glucocorticoid receptors (GRs) exert essential immunoregulatory and anti-inflammatory actions, the interaction between GRs and NF- $\mathrm{kB}$ signaling is of particular importance. In principle, GR signaling and NF- $\mathrm{BB}$ signaling are mutually antagonistic. Dysregulated GR signaling may enable exaggerated NF- $\kappa B$ signaling, ultimately leading to greater inflammatory responses. In the current study, a decreased expression of GR in MS rats was observed, and MS rats had a greater percentage of DNA methylation in the exon $1_{7}$ GR promoter region (Fig. 5). These findings are in agreement with those of other studies that showed that early life adversity results in greater methylation of the GR gene promoter and reduced GR expression in rodents and humans, with consequent increases in pro-inflammatory gene expression [29-34]. Therefore, we speculate that the MS phenotype was associated with the downregulation of
GR and the increased production of pro-inflammatory cytokines controlled by the pro-inflammatory transcription factor NF-kB, which may worsen sevoflurane anesthesia-induced neuroinflammation and ultimately lead to cognitive impairment. A key question is whether the impact of early experiences on epigenetic programming is reversible in adults. It has been reported that DNA methylation produced by insufficient maternal care is reversible in the hippocampus of adult offspring through the modulation of the chromatin structure using the histone deacetylase inhibitor TSA [50]. Histone deacetylase prevents histone acetylation and ensures that histones bind tightly to DNA. The activation of chromatin through histone deacetylase inhibition might trigger DNA demethylation by increasing the accessibility of methylated DNA to demethylase activity [51]. We found that TSA decreased the percentage of 

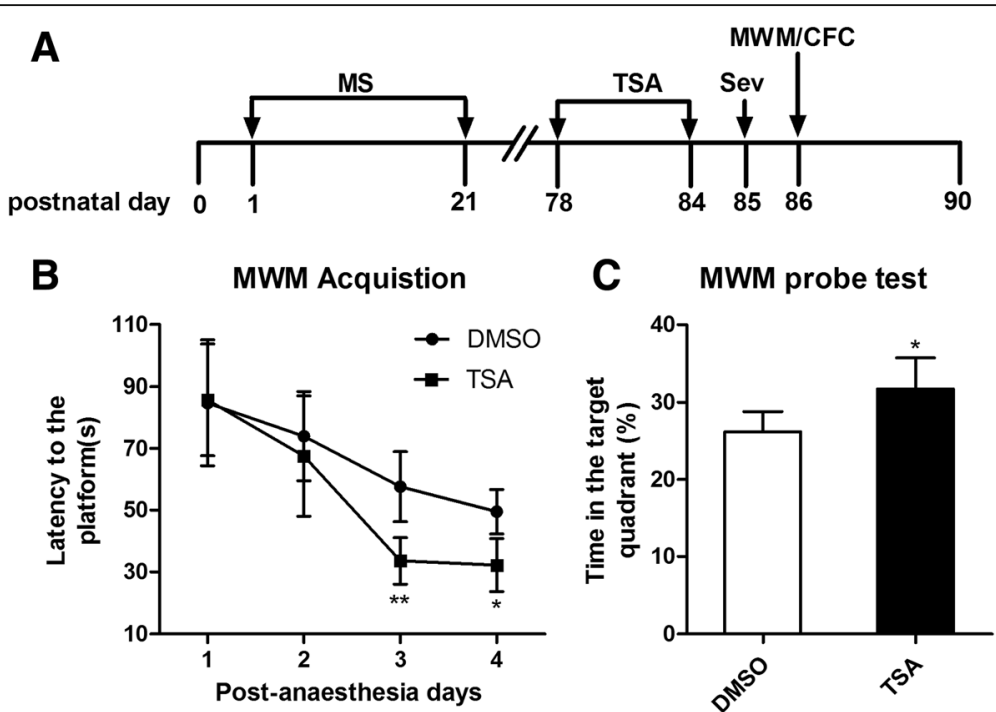

C MWM probe test
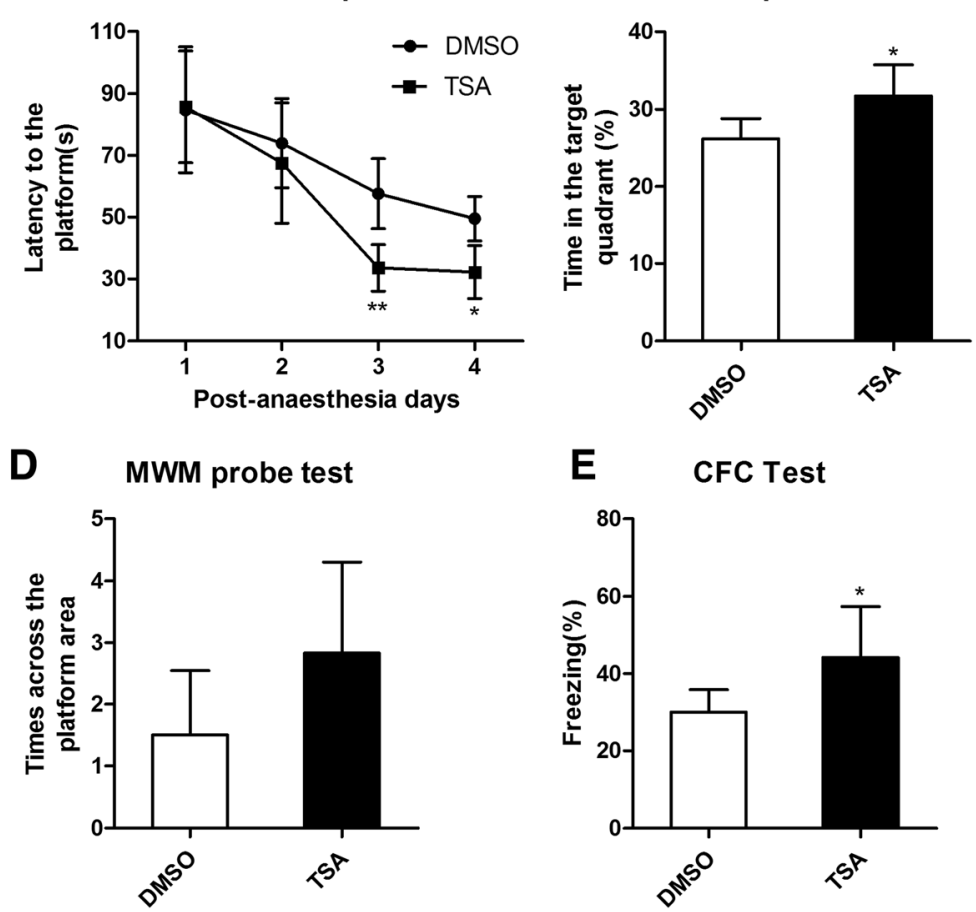

Fig. 8 TSA attenuated sevoflurane-induced cognitive impairment in adult rats with neonatal maternal separation. a Schematic illustration of the experimental timeline. MS maternal separation, Sev anesthesia with $3 \%$ sevoflurane for $2 \mathrm{~h}$, TSA trichostatin A, MWM Morris water maze, CFC context fear conditioning. $\mathbf{b}$ TSA group rats had a shorter escape latency than DMSO group rats on the third and fourth days after sevoflurane anesthesia. There was a statistically significant interaction of time and group between the different groups ( $\left.{ }^{*} P<0.05\right)$. Analysis was performed by repeated-measures two-way ANOVA. c The time in the target quadrant in the TSA group was more than that in the DMSO group by the MWM probe test. $\mathbf{d}$ There was no significant difference in the number of crossings of the platform area between the two groups of rats. e TSA group rats exhibited increased freezing to context relative to DMSO group rats. ${ }^{*} P<0.05,{ }^{* *} P<0.01$ versus DMSO. Error bars represent the means \pm SD $(n=10)$. Statistical analyses were performed with Student's two-sample $t$ test

DNA methylation in the exon $1_{7}$ GR promoter region. Then, marked increases in the hippocampal GR expression were accompanied by the reversal of DNA methylation (Fig. 6).

Finally, we assessed the effects of the reversal of GR expression in adult MS rats on the activation of NF- $\mathrm{kB}$ signaling and neuroinflammatory responses to sevoflurane anesthesia. TSA pretreatment significantly suppressed overactivated NF- $\mathrm{KB}$ signaling and excessive neuroinflammation (Fig. 7). Moreover, TSA pretreatment ameliorated sevoflurane anesthesia-induced cognitive impairment in adult MS rats. These results suggested that enhanced GR expression may suppress the inflammatory response and prevent cognitive impairment induced by sevoflurane anesthesia. Taken together, our findings support the conclusion that early life adversity downregulates the expression of GR, enhances NF- $\mathrm{kB}$ signaling, and worsens sevoflurane anesthesia-induced neuroinflammation, ultimately leading to cognitive impairment.

There are several limitations and caveats that should be considered in this study. First, we did not systematically investigate the effects of MS on the function of the hypothalamic-pituitary-adrenal (HPA) axis and instead focused on the expression of GR in the hippocampus, which is closely linked to HPA axis function [52]. It is worth noting that higher cortisol levels and ineffective dexamethasone treatments have been reported in POCD patients [53, 54]. However, the current results suggested that the MS-induced downregulation of GR expression in the hippocampus may amplify neuroinflammation and cause cognitive impairment, which will allow us to further investigate the association between early life 
adversity, the HPA axis, and POCD. Second, MS can induce DNA hypermethylation in other genes (e.g., brain-derived neurotrophic factor) in addition to the GR gene, and the demethylation function of TSA is not specific to the GR gene $[55,56]$. More research is needed to determine whether other mechanisms of early life adversity are involved in cognitive impairment after anesthesia. However, given the inflammatory mechanisms of POCD, the epigenetic regulation of GR is most likely the chief reason for MS-induced cognitive impairment after anesthesia. Finally, volatile anesthetics seem to have a confounding effect on the brain. Some beneficial effects have been reported for isoflurane on the outcome of cerebral ischemia-reperfusion injury, traumatic brain injury, and lipopolysaccharide-induced cerebral damage [57-59]. Perhaps volatile anesthetics have a neuroprotective effect on brain injury. However, volatile anesthetics themselves may have adverse effects on the brain. Recent studies show that volatile anesthetics can cause neuroinflammation and impairment in a normal physiological state $[10,11]$.

\section{Conclusions}

We found that early life adversity may be associated with increased vulnerability to cognitive decline after anesthesia. These findings suggest that the downregulation of GR by MS enhanced NF- $\mathrm{KB}$ signaling and sevoflurane anesthesiainduced neuroinflammation, which plays an important role in cognitive impairment. In addition, the regulation of GR expression in the hippocampus may be one strategy to prevent anesthesia-induced cognitive impairment under certain conditions. This study provides insight into the mechanisms by which early life adversity produces a future risk of POCD. In future studies, it will be important to further determine whether early life adversity is an independent risk factor for POCD in clinical practice, ultimately leading to safer anesthesia and better postoperative prognoses for people who have experienced early life adversity.

\section{Abbreviations \\ CFC: Context fear conditioning; ELISA: Enzyme-linked immunosorbent assay; GFAP: Glial fibrillary acidic protein; GR: Glucocorticoid receptor;

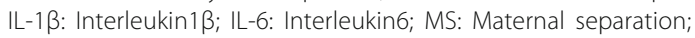 MWM: Morris water maze; NF-kB: Nuclear factor-kappa B; PDTC: Pyrrolidine dithiocarbamate; POCD: Postoperative cognitive dysfunction; TNF-a: Tumor necrosis factor-a; TSA: Trichostatin A}

\section{Acknowledgements}

We thank Nan Sun and Peng Wu (Jiangsu Province Key Laboratory of Anesthesiology, Xuzhou Medical University, Xuzhou, China) for the assistance with the experimental instruction and Ke Wang (Department of Statistics, Xuzhou Medical University, Xuzhou, China) for the assistance with the statistical analysis.

\section{Funding}

This work was supported by the National Natural Science Foundation of China (No. 81171013) and the Natural Science Foundation of Jiangsu Province (No. BK20151169).

\section{Availability of data and materials}

All data generated or analyzed during this study are included in this published article.

\section{Authors' contributions}

YZZ formulated the design of the studies, carried out the execution and analysis of the studies, and drafted the manuscript. YW, RY, TH, and HH performed the research and the statistical analysis. JLC participated in formulating the design of the study. LWW participated in the study's design and coordination and helped to draft the manuscript. YQW conceived the study, completed its design and coordination, and secured funding for the project. All authors read and approved the final manuscript.

\section{Competing interests}

The authors declare that they have no competing interests.

\section{Consent for publication}

Not applicable.

\section{Ethics approval}

All animals were treated in accordance with the International Guidelines for animal research. All experimental procedures involving animals were approved by the Animal Care and Use Committee of Xuzhou Medical University.

\section{Author details}

'Jiangsu Province Key Laboratory of Anesthesiology, Xuzhou Medical University, 209 Tongshan, Xuzhou 221004, People's Republic of China. ${ }^{2}$ Department of Anesthesiology, Xuzhou Central Hospital, 199 Jiefang South Road, Xuzhou, People's Republic of China. ${ }^{3}$ Department of Anesthetic Pharmacology, Xuzhou Medical University, Xuzhou, People's Republic of China.

Received: 29 September 2016 Accepted: 20 December 2016

Published online: 07 January 2017

\section{References}

1. Steinmetz J, Christensen KB, Lund T, Lohse N, Rasmussen LS. Long-term consequences of postoperative cognitive dysfunction. Anesthesiology. 2009;110:548-55.

2. Monk TG, Weldon BC, Garvan CW, Dede DE, van der Aa MT, Heilman KM, et al. Predictors of cognitive dysfunction after major noncardiac surgery. Anesthesiology. 2008;108:18-30.

3. Terrando N, Eriksson LI, Ryu JK, Yang T, Monaco C, Feldmann M, et al. Resolving postoperative neuroinflammation and cognitive decline. Ann Neurol. 2011;70:986-95.

4. Peng L, Xu L, Ouyang W. Role of peripheral inflammatory markers in postoperative cognitive dysfunction (POCD): a meta-analysis. PLoS One. 2013;8:e79624.

5. Vacas S, Degos V, Feng X, Maze M. The neuroinflammatory response of postoperative cognitive decline. Br Med Bull. 2013;106:161-78.

6. Rosczyk H, Sparkman NL, Johnson R. Neuroinflammation and cognitive function in aged mice following minor surgery. Exp Gerontol. 2008;43:840-6.

7. Terrando N, Monaco C, Ma D, Foxwell BM, Feldmann M, Maze M. Tumor necrosis factor-alpha triggers a cytokine cascade yielding postoperative cognitive decline. Proc Natl Acad Sci U S A. 2010;107:20518-22.

8. Cibelli M, Fidalgo AR, Terrando N, Ma D, Monaco C, Feldmann M, et al. Role of interleukin-1 beta in postoperative cognitive dysfunction. Ann Neurol. 2010;68:360-8.

9. Tan H, Cao J, Zhang J, Zuo Z. Critical role of inflammatory cytokines in impairing biochemical processes for learning and memory after surgery in rats. J Neuroinflammation. 2014;11:93.

10. Wu X, Lu Y, Dong Y, Zhang G, Zhang Y, Xu Z, et al. The inhalation anesthetic isoflurane increases levels of proinflammatory TNF-alpha, IL-6, and IL-1 beta. Neurobiol Aging. 2012;33:1364-78.

11. Culley DJ, Baxter MG, Yukhananov R, Crosby G. Long-term impairment of acquisition of a spatial memory task following isoflurane-nitrous oxide anesthesia in rats. Anesthesiology. 2004;100:309-14. 
12. Culley DJ, Baxter MG, Crosby CA, Yukhananov R, Crosby G. Impaired acquisition of spatial memory 2 weeks after isoflurane and isoflurane-nitrous oxide anesthesia in aged rats. Anesth Analg. 2004;99:1393-7.

13. Moller JT, Cluitmans P, Rasmussen LS, Houx P, Rasmussen H, Canet J, et al. Long-term postoperative cognitive dysfunction in the elderly ISPOCD1 study. ISPOCD investigators. International study of post-operative cognitive dysfunction. Lancet. 1998:351:857-61.

14. Cortese GP, Barrientos RM, Maier SF, Patterson SL. Aging and a peripheral immune challenge interact to reduce mature brain-derived neurotrophic factor and activation of TrkB, PLCgamma1, and ERK in hippocampal synaptoneurosomes. J Neurosci. 2011;31:4274-9.

15. Dilger RN, Johnson RW. Aging, microglial cell priming, and the discordant central inflammatory response to signals from the peripheral immune system. J Leukoc Biol. 2008;84:932-9.

16. Barrientos RM, Hein AM, Frank MG, Watkins LR, Maier SF. Intracisternal interleukin-1 receptor antagonist prevents postoperative cognitive decline and neuroinflammatory response in aged rats. J Neurosci. 2012:32:14641-8.

17. Beloosesky Y, Hendel D, Weiss A, Hershkovitz A, Grinblat J, Pirotsky A, et al. Cytokines and C-reactive protein production in hip-fracture-operated elderly patients. J Gerontol A Biol Sci Med Sci. 2007;62:420-6.

18. Hovens IB, Schoemaker RG, van der Zee EA, Absalom AR, Heineman $E$, van Leeuwen BL. Postoperative cognitive dysfunction: involvement of neuroinflammation and neuronal functioning. Brain Behav Immun. 2014;38:202-10.

19. Norman RE, Byambaa M, De R, Butchart A, Scott J, Vos T. The long-term health consequences of child physical abuse, emotional abuse, and neglect: a systematic review and meta-analysis. PLoS Med. 2012;9:e1001349.

20. Chapman DP, Whitfield CL, Felitti VJ, Dube SR, Edwards VJ, Anda RF. Adverse childhood experiences and the risk of depressive disorders in adulthood. J Affect Disord. 2004:82:217-25.

21. Anda RF, Dong M, Brown DW, Felitti VJ, Giles WH, Perry GS, et al. The relationship of adverse childhood experiences to a history of premature death of family members. BMC Public Health. 2009;9:106.

22. Dube SR, Fairweather D, Pearson WS, Felitti VJ, Anda RF, Croft JB. Cumulative childhood stress and autoimmune diseases in adults. Psychosom Med. 2009;71:243-50.

23. Brown DW, Anda RF, Felitti VJ, Edwards VJ, Malarcher AM, Croft JB, et al. Adverse childhood experiences are associated with the risk of lung cancer: a prospective cohort study. BMC Public Health. 2010;10:20.

24. Rich-Edwards JW, Spiegelman D, Hibert ENL, Jun HJ, Todd TJ, Kawachi I, et al. Abuse in childhood and adolescence as a predictor of type 2 diabetes in adult women. Am J Prev Med. 2010;39:529-36.

25. Riley EH, Wright RJ, Jun HJ, Hibert EN, Rich-Edwards JW. Hypertension in adult survivors of child abuse: observations from the nurses' health study II. J Epidemiol Community Health. 2010;64:413-8.

26. Kittleson MM, Meoni LA, Wang NY, Chu AY, Ford DE, Klag MJ. Association of childhood socioeconomic status with subsequent coronary heart disease in physicians. Arch Intern Med. 2006;166:2356-61.

27. Keinan-Boker L, Vin-Raviv N, Liphshitz I, Linn S, Barchana M. Cancer incidence in Israeli Jewish survivors of World War II. J Natl Cancer Inst. 2009; 101:1489-500.

28. Miller GE, Chen E, Fok AK, Walker H, Lim A, Nicholls EF, et al. Low early-life social class leaves a biological residue manifested by decreased glucocorticoid and increased proinflammatory signaling. Proc Natl Acad Sci U S A. 2009;106:14716-21.

29. Weaver IC, Cervoni N, Champagne FA, D'Alessio AC, Sharma S, Seckl JR, et al. Epigenetic programming by maternal behavior. Nat Neurosci. 2004;7:847-54.

30. Tyrka AR, Price LH, Marsit C, Walters OC, Carpenter LL. Childhood adversity and epigenetic modulation of the leukocyte glucocorticoid receptor: preliminary findings in healthy adults. PLoS One. 2012;7:e30148.

31. McGowan PO, Sasaki A, D'Alessio AC, Dymov S, Labonte B, Szyf M, et al. Epigenetic regulation of the glucocorticoid receptor in human brain associates with childhood abuse. Nat Neurosci. 2009;12:342-8.

32. Ladd CO, Huot RL, Thrivikraman KV, Nemeroff CB, Plotsky PM. Long-term adaptations in glucocorticoid receptor and mineralocorticoid receptor mRNA and negative feedback on the hypothalamo-pituitary-adrenal axis following neonatal maternal separation. Biol Psychiatry. 2004;55:367-75.

33. Miller GE, Chen E. Harsh family climate in early life presages the emergence of a proinflammatory phenotype in adolescence. Psychol Sci. 2010;21:848-56.
34. Gouin JP, Glaser R, Malarkey WB, Beversdorf D, Kiecolt-Glaser JK. Childhood abuse and inflammatory responses to daily stressors. Ann Behav Med. 2012:44:287-92.

35. Levine ME, Cole SW, Weir DR, Crimmins EM. Childhood and later life stressors and increased inflammatory gene expression at older ages. Soc Sci Med. 2015;130:16-22.

36. Slopen N, Loucks EB, Appleton AA, Kawachi I, Kubzansky LD, Non AL, et al. Early origins of inflammation: an examination of prenatal and childhood social adversity in a prospective cohort study. Psychoneuroendocrinology. 2015;51:403-13.

37. Avitsur R, Hunzeker J, Sheridan JF. Role of early stress in the individual differences in host response to viral infection. Brain Behav Immun. 2006:20:339-48

38. Rodriguez DAL, Duenas Z. Maternal separation during breastfeeding induces gender-dependent changes in anxiety and the GABA-A receptor alpha-subunit in adult wistar rats. PLoS One. 2013;8:e68010.

39. Li ZQ, Rong XY, Liu YJ, Ni C, Tian XS, Mo N, et al. Activation of the canonical nuclear factor-kappaB pathway is involved in isoflurane-induced hippocampal interleukin-1beta elevation and the resultant cognitive deficits in aged rats. Biochem Biophys Res Commun. 2013;438:628-34.

40. Tian Y, Guo S, Wu X, Ma L, Zhao X. Minocycline alleviates sevoflurane-induced cognitive impairment in aged rats. Cell Mol Neurobiol. 2015;35:585-94.

41. Zhang XH, Wu LJ, Gong B, Ren M, Li BM, Zhuo M. Induction- and conditioning-protocol dependent involvement of NR2B-containing NMDA receptors in synaptic potentiation and contextual fear memory in the hippocampal CA1 region of rats. Mol Brain. 2008;1:9.

42. Logue SF, Paylor R, Wehner JM. Hippocampal lesions cause learning deficits in inbred mice in the Morris water maze and conditioned-fear task. Behav Neurosci. 1997;111:104-13.

43. Shuman T, Wood SC, Anagnostaras SG. Modafinil and memory: effects of modafinil on Morris water maze learning and pavlovian fear conditioning. Behav Neurosci. 2009;123:257-66.

44. Zhang L, Zhang J, Yang L, Dong Y, Zhang Y, Xie Z. Isoflurane and sevoflurane increase interleukin-6 levels through the nuclear factor-kappa B pathway in neuroglioma cells. Br J Anaesth. 2013;110:182-91.

45. Bracchi-Ricard V, Lambertsen KL, Ricard J, Nathanson L, Karmally S, Johnstone J, et al. Inhibition of astroglial NF-kappaB enhances oligodendrogenesis following spinal cord injury. J Neuroinflammation. 2013;10:92.

46. Morishita S, Oku H, Horie T, Tonari M, Kida T, Okubo A, et al. Systemic simvastatin rescues retinal ganglion cells from optic nerve injury possibly through suppression of astroglial NF-kB activation. PLoS One. 2014;9:e84387.

47. Bernardino L, Xapelli S, Silva AP, Jakobsen B, Poulsen FR, Oliveira CR, et al. Modulator effects of interleukin-1 beta and tumor necrosis factor-alpha on AMPA-induced excitotoxicity in mouse organotypic hippocampal slice cultures. J Neurosci. 2005;25:6734-44.

48. Vallabhapurapu S, Karin M. Regulation and function of NF-kappaB transcription factors in the immune system. Annu Rev Immunol. 2009;27:693-733.

49. Barnes PJ, Karin M. Nuclear factor-kappaB: a pivotal transcription factor in chronic inflammatory diseases. N Engl J Med. 1997;336:1066-71.

50. Roth SY, Denu JM, Allis CD. Histone acetyltransferases. Annu Rev Biochem. 2001;70:81-120.

51. Cervoni N, Szyf M. Demethylase activity is directed by histone acetylation. J Biol Chem. 2001;276:40778-87.

52. Bratt AM, Kelley SP, Knowles JP, Barrett J, Davis K, Davis M, et al. Long term modulation of the HPA axis by the hippocampus. Behavioral, biochemical and immunological endpoints in rats exposed to chronic mild stress. Psychoneuroendocrinology. 2001;26:121-45.

53. Ji MH, Shen JC, Gao R, Liu XY, Yuan HM, Dong L, et al. Early postoperative cognitive dysfunction is associated with higher cortisol levels in aged patients following hip fracture surgery. J Anesth. 2013;27:942-4.

54. Ottens TH, Dieleman JM, Sauer AM, Peelen LM, Nierich AP, de Groot WJ, et al. Effects of dexamethasone on cognitive decline after cardiac surgery: a randomized clinical trial. Anesthesiology. 2014;121:492-500.

55. Anier K, Malinovskaja K, Pruus K, Aonurm-Helm A, Zharkovsky A, Kalda A. Maternal separation is associated with DNA methylation and behavioural changes in adult rats. Eur Neuropsychopharmacol. 2014;24:459-68.

56. Roth TL, Lubin FD, Funk AJ, Sweatt JD. Lasting epigenetic influence of early-life adversity on the BDNF gene. Biol Psychiatry. 2009;65:760-9. 
57. Wang JK, Yu LN, Zhang FJ, Yang MJ, Yu J, Yan M, et al. Postconditioning with sevoflurane protects against focal cerebral ischemia and reperfusion injury via PI3K/Akt pathway. Brain Res. 2010;1357:142-51.

58. Statler $K D$, Alexander $H$, Vagni $V$, Dixon $C E$, Clark RS, Jenkins $L$, et al. Comparison of seven anesthetic agents on outcome after experimental traumatic brain injury in adult, male rats. J Neurotrauma. 2006;23:97-108.

59. Xu X, Kim JA, Zuo Z. Isoflurane preconditioning reduces mouse microglial activation and injury induced by lipopolysaccharide and interferon-gamma. Neuroscience. 2008;154:1002-8

Submit your next manuscript to BioMed Central and we will help you at every step:

- We accept pre-submission inquiries

- Our selector tool helps you to find the most relevant journal

- We provide round the clock customer support

- Convenient online submission

- Thorough peer review

- Inclusion in PubMed and all major indexing services

- Maximum visibility for your research

Submit your manuscript at www.biomedcentral.com/submit
Biomed Central 